www.vum.nomos.de

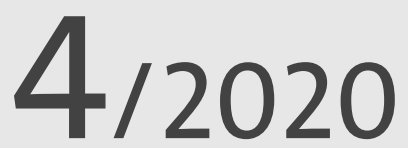

26. Jahrgang, Seiten 153-208

Herausgeber: Univ.-Prof. em. Dr. Heinrich Reinermann, Deutsche Universität für Verwaltungswissenschaften Speyer | Univ.-Prof. Dr. Veith Mehde, Mag.rer.publ., Leibniz Universität Hannover (geschäftsführend) | Prof. Dr. Tino Schuppan, Hochschule der Bundesagentur für Arbeit, Schwerin (geschäftsführend)

Beirat: Helmut Dedy, Geschäftsführendes Präsidialmitglied des Deutschen Städtetages, Köln | Dr. Hans Bernhard Beus, Staatssekretär a.D. im Bundesministerium der Finanzen, Berlin | Prof. Dr. Martin Brüggemeier, Hochschule für Technik und Wirtschaft, Berlin | Hans Jörg Duppré, Landrat, Präsident des Deutschen Landkreistages, Berlin | Univ.-Prof. Dr. Gisela Färber, Deutsche Universität für Verwaltungswissenschaften Speyer | Prof. Dr. Gerhard Hammerschmid, Hertie School of Governance GmbH, Berlin | Peter Heesen, Bundesvorsitzender des Deutschen Beamtenbundes, Bonn | Dr. Gerd Landsberg, Geschäftsführendes Präsidialmitglied des Deutschen Städte- und Gemeindebundes, Berlin | Prof. Dr. Andreas Lasar, Hochschule Osnabrück | Dr. Johannes Meier, Mitglied des Vorstands der Bertelsmann-Stiftung, Gütersloh | Univ.-Prof. Dr. Isabella Proeller, Universität Potsdam | Prof. Dr. Marga Pröhl, Generaldirektorin des European Institute of Public Administration (EIPA), Maastricht | Dr. Sebastian Saxe, Mitglied der Geschäftsleitung der Hamburg Port Authority Anstalt des öffentlichen Rechts, Hamburg | Univ.-Prof. Dr. Christina Schaefer, Helmut Schmidt Universität, Hamburg | Kay Scheller, Präsident des Bundesrechnungshofes, Bonn | Prof. Dr. Reto Steiner, ZHAW School of Management and Law, Winterthur | Prof. Dr. Arthur Winter, Donau-Universität Krems | Christian Zahn, Mitglied des Bundesvorstands der Vereinten Dienstleistungsgewerkschaft ver.di, Berlin

\title{
Bleib innovativ, Verwaltung!
}

Hermann Hill

Das Thema Innovation wird auch für Verwaltungen immer spannender. Die Verknüpfung mit den Themen Nachhaltigkeit, Wahrnehmung (Kognition) und Design zeigt neue Perspektiven. Zudem liefert der Beitrag konkrete Hinweise für Innovationsworkshops sowie Innovationskommunikation und -transfer.

\section{Von isolierten Neuansätzen zu nachhaltiger Innovation}

Verwaltungsmodernisierung, innovatives Verwaltungshandeln und generell Change Management ${ }^{1}$ stellen keinen Sprung von einem Zustand A in einen Zustand B dar. Vielmehr sind angesichts ständiger neuer Herausforderungen, etwa durch dynamische Entwicklungen in der Digitalisierung, eine grundsätzliche Innovationshaltung sowie eine beständige Innovationspraxis

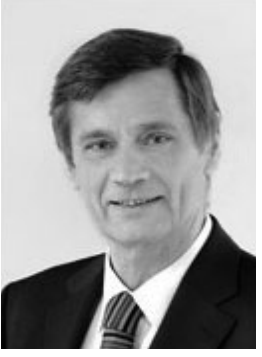

\section{Univ.-Prof. Dr.} Hermann Hill Vertretung eines Lehrstuhls an de Deutschen Universität für Verwaltungswissenschaften, Speyer. gefragt. So ist etwa mit der Einführung einer elektronischen Akte die digitale Transformation nicht abgeschlossen, ebenso stellt die Gründung eines Innovation Labs oder die Integration eines Start-ups ${ }^{2}$ nicht das Ende eines Innovationsprozesses dar. Nach „Sei innovativ, Verwaltung!" 3 gilt daher „Bleib innovativ, Verwaltung!“

Das Hauptgutachten des Wissenschaftlichen Beirats der Bundesregierung Globale Umweltveränderungen (WBGU) „Unsere gemeinsame digitale Zukunft" ${ }^{4}$ stellt zurecht Innovation, Digitalisierung und Nachhaltigkeit in einen Zusammenhang und fordert eine (transformative) „Bildung für die digitalisierte Nachhaltigkeitsgesellschaft"5. Die Potenziale der Digitalisierung sollten für Nachhaltigkeit und Wohlergehen des Menschen genutzt werden. Es gehe um „verantwortungsvolle Innovation“, bei der sowohl Zielbestimmungen als auch mögliche $\mathrm{Ri}$ - siken für Nachhaltigkeit von vorneherein mitbedacht werden ${ }^{6}$.

Das Gutachten verweist zudem auf die Risiko- und Resilienzforschung, die sich mit Nichtwissen und Nichtlinearität in Systemen befasse ${ }^{7}$. In diesem Kontext gilt: Innovationsversuche können auch fehlschlagen, ja teilweise wird das Startup-Prinzip „Fail often, fail early“ sogar als Grundregel innovativen Handelns bezeichnet ${ }^{8}$. Innovationen sind zudem fragil und fluide, können in dynamischen Kontexten schnell wieder überholt sein. Insbesondere wenn im Zusammenhang mit Innovation und Digitalisierung ein agiles Vorgehen empfohlen wird, liegt es daher nahe, Agilität mit Resilienz zu verknüp-

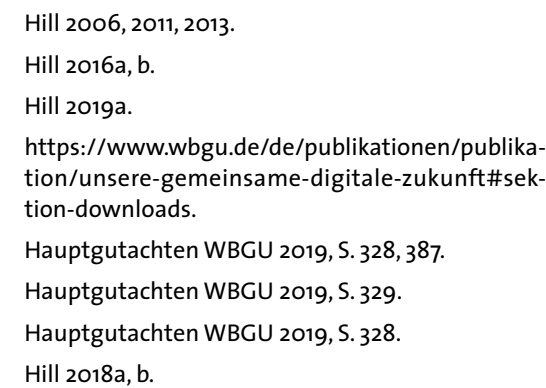
tion/unsere-gemeinsame-digitale-zukunft\#sektion-downloads.

Hauptgutachten WBGU 2019, S. 328, 387.

Hauptgutachten WBGU 2019, S. 329.

Hauptgutachten WBGU 2019, S. 328.

Hill 2018a, b. 
fen. ${ }^{9}$ Das mit Resilienz (Widerstands- und Anpassungsfähigkeit) verbundene Konzept der Salutogenese ${ }^{10}$ empfiehlt, trotz Vorhandenseins von Gefahren und Missbräuchen das Positive zu sehen und aktiv voranzuschreiten und damit insgesamt eine Stimmigkeit (Kohärenz) anzustreben.

Teilweise wird versucht, Innovationsfähigkeit, Innovationsbereitschaft und Innovationserfolg durch Indikatoren zu mes$\operatorname{sen}^{11}$. Solange man sich nicht sklavisch auf die Zahlen fixiert und das Mittel zum Zweck macht, sondern auch die Ziele und den breiteren Kontext im Auge behält ${ }^{12}$, bildung und Erfahrung haben und in gleicher Weise denken, entsteht selten etwas Neues. Innovationen entstehen an Schnittstellen. Das heißt für eine nachhaltige Innovation aber auch, dass die Mitglieder des Innovationsteams häufiger wechseln oder zumindest kritische Impulse von außen gesucht werden sollten.

Dieser Impulse und Motivationsanreize (challenge) bedarf es auch für eine nachhaltige Innovationsbereitschaft. Der vielzitierte „Stillstand ist Rückschritt“, Ausruhen auf Erfolgen gilt nicht, zumal auch die Herausforderungen sich ständig

\section{"Angesichts ständiger neuer Heraus- forderungen, etwa durch dynamische Entwicklungen in der Digitalisierung, sind eine grundsätzliche Innovationshaltung sowie eine beständige Innovationspraxis gefragt. Ausruhen auf Erfolgen gilt nicht."}

mag dies ein Weg sein, um sich durch Reflektion und zukunftsgerichtete Evaluation den Weg zu nachhaltigem Innovationsverhalten bewusst zu machen.

Wie kann nachhaltige Innovation gelingen? Das World Economic Forum hat in seinem Bericht 2016 eine „kognitive Flexibilität“ als eine der zehn wichtigsten Fähigkeiten für die Zukunft bezeichnet. ${ }^{13}$ Darunter kann man die Fähigkeit verstehen, unsere Gedanken zwischen verschiedenen Konzepten oder Perspektiven hin und her zu bewegen. ${ }^{14}$ Dies unterscheidet uns von der künstlichen Intelligenz der Maschinen. Nach Bernhard Schölkopf ist das Interessante an unserer Intelligenz, dass wir Go spielen können und dann vom Tisch aufstehen und Essen machen können, was eine Maschine (noch) nicht kann. ${ }^{15}$ Deswegen wird künstliche Intelligenz auch als „digitaler Fachidiot“ bezeichnet. $^{16}$

Zur kognitiven Flexibilität (geistigen Beweglichkeit) kommt etwa in Innovation Labs das Erfordernis einer „kognitiven Diversität“"17. Wenn alle die gleiche Aus- wandeln. Damit verbunden ist deshalb eine „Volatilitätsvorsorge“, ein Frühwarnsystem, das ständig versucht, neue Trends $\mathrm{zu}$ identifizieren, $\mathrm{zu}$ analysieren und auf ihre Relevanz für die Organisation abzuprüfen.

Auch dies sollte nicht durch eine Person geschehen, sondern „cross-funktional“, um Wechselwirkungen und Zusammenhänge nicht zu übersehen. Co-Evolution ist das vielzitierte Stichwort. ${ }^{18}$ Damit andere angeregt werden und ihre Ideen einbringen können, bieten sich Innovationsplattformen an, die Transparenz und Austausch ermöglichen. Schließlich sind regelmäßige Vergewisserungen über den Zweck (purpose) und den gewünschten Impact der Innovationen erforderlich.

\section{Kognitive Herausforderungen für Lernen und Innovation}

Der wissenschaftliche Beirat Globale Umweltveränderungen wies in seinem zitierten Gutachten darauf hin, wie Menschen Wirklichkeit wahrnehmen und verstehen, beeinflusse, wie sie sie erleben und bewer- ten und damit auch, welche unternehmerischen und politischen Maßnahmen sie für sinnvoll, machbar und legitim hielten. Dies bestimme die Innovationsfähigkeit, Diskursfähigkeit, Gestaltungsfähigkeit und Handlungsfähigkeit einer Gesellschaft. ${ }^{19}$

Die Prozesse der Wahrnehmung sind daher entscheidend für weiteres (innovatives) Handeln. Psychologie und Hirnforschung haben jedoch schon länger darauf hingewiesen, dass Menschen auf Täuschungen und Fallen hereinfallen und Fehler machen. Dabei gibt es sowohl statistische Fallen bei der Datenanalyse (Data Bias) als auch optische Täuschungen, die zu grundlegenden Fehlinterpretationen führen. Denkblockaden, emotionale Verfärbung oder kulturelle Muster tun ein Übriges. Auch in Gruppen und Teams sind dysfunktionale Einflüsse bekannt. Subtile Einflussmechanismen (Nudging, Priming, Framing, Microtargeting) sind darauf angelegt, ein bestimmtes Verhalten bei uns zu erzeugen. ${ }^{20}$

Hinzu kommen die Besonderheiten der digitalen Welt. Tristan Harris hat kürzlich in der New York Times unter Hinweis auf Edward O. Wilson darauf hingewiesen, dass den steinzeitlichen Impulsen unseres Gehirns gottgleiche Technologien gegenüberstünden. Mit diesem Denken seien wir einfach unfähig, den „Geschenken“ der Technologiekonzerne zu widerstehen. ${ }^{21}$

Dabei geht es nicht nur um das Einsammeln und Ausnutzen unserer Daten, um sog. Köder (Clickbaits) oder verfüh-

\footnotetext{
Reinhardt 2018

Hill 2014, S. 93, Roth 2019, S. 96.

Kreimeier 2017, 2018.

Rettig 2020.

https://www.weforum.org/agenda/2016/o1/the10-skills-you-need-to-thrive-in-the-fourth-industrial-revolution/; Rogl 2019, S. 222.

14 https://coreaxis.com/top-skills-future-enhancecognitive-flexibility/.

15 FAZ vom 26. Februar 2020, S. 20.

16 Borth 2019, S. 139

Hill 2020, S. 207

Bormann, u.a. 2019; Schlumpf 2019.

9 Hauptgutachten WBGU 2019, S. 328.

20 Ausführlich Hill 2020, S. $208 \mathrm{ff}$.

21 Harris 2019
} 
rerische Muster (Dark Patterns), sondern auch um gezielte Desinformationen, wie Fake News oder Deep Fakes oder das nicht ersichtliche Handeln von Social Bots bis hin zu Tunnelvisionen oder Diskriminierung durch Algorithmen.

Zunehmend wird daher an Methoden zur Bewusstmachung subtiler Einflüsse oder Aufdeckung von Fehlinformationen (Faktenfinder) sowie an verbesserten Fähigkeiten zum Umgang mit Daten gearbeitet. Hinzu kommen Strategien für Lernen und Innovation. Trotz „Bounded Rationality“ (eingeschränkter Rationalität) handlungsfähig zu bleiben ist dabei das Ziel. ${ }^{22}$

Vielleicht kann uns auch dabei künstliche Intelligenz behilflich sein. Durch Such- und Auswertungsalgorithmen können dem Menschen außergewöhnliche, auf Daten gestützte Erkenntnisse verschafft werden. Sprachgesteuerte Interaktionen ermöglichen gemeinsames Lernen. In konkreten physischen Räumen lassen sich neue Formen der Kollaboration entwickeln.

Auf diese Weise entstünden Mitarbeiter mit Superfähigkeiten. Wenn sie künstliche Intelligenz im Wissen um ihre Fähigkeiten, aber auch Grenzen einsetzten, könne dies zu neuen Denkansätzen und Geschäftsmodellen führen. ${ }^{23}$

Darüber hinaus lernen Algorithmen zunehmend auch, mit Unschärfe umgehen zu können und pragmatische Lösungen $\mathrm{zu}$ entwickeln. Durch zufallsgesteuerte Abweichungen oder bewusste Fehlversuche entstehen Anpassungsleistungen und neue Wege zur Problemlösung. ${ }^{24}$

\section{Innovations-Design}

Kognitionsforscher weisen darauf hin, dass das Gehirn die Wirklichkeit bestimme. Sensorische Information seien mehrdeutig und ungenau, weshalb sie keine hinreichende Basis für unsere Wahrnehmung darstellten. Der Organismus mache unbewusst Vorannahmen, die zur Analyse der eingehenden Sinnesinformationen beisteuerten ${ }^{25}$. Ebenso heißt es an anderer Stelle, Wahrnehmung beruhe auf Vorhersagen, die das Gehirn über die Ursachen eintreffender Sinnessignale anstelle. Durch
Abweichungen von diesen Prognosen korrigierten wir laufend unser Weltbild. Das Gehirn entwickle und aktualisiere plausible Hypothesen über die Sinneseindrücke. $^{26}$

Dies erinnert an klassische Verständnisse eines Konstruktivismus, bei dem der Beobachter sich die Welt erschafft. Sog. Kippbilder, wie etwa Hase und Vogel, oder die Wahrnehmung von Mustern in Wolken, am Sternenhimmel oder in Tintenklecksen bestätigen, dass es vom Betrachter abhängt, was er erkennt. Dabei knüpfe, das uns wichtig sei und uns persönlich angehe. ${ }^{29}$ Möglicherweise kann auch die in modernen Innovationsprozessen empfohlene radikale Nutzerorientierung $^{30}$ in dieser Richtung wirken, indem die Mitwirkenden am Innovationsprozess etwas Sinnvolles, Weiterführendes, also Gutes für die Nutzer tun wollen.

Daraus lassen sich verschiedene Strategien für das Innovations-Design gewinnen. Wichtig ist zunächst das Umfeld. Es richtet den Assoziationsraum aus und erweitert ihn. Es verändere auch unser

\section{"Radikale Nutzerorientierung, indem die Mitwirkenden am Innovationsprozess etwas Sinnvolles, Weiterführendes, also Gutes für die Nutzer tun wollen."}

ist nachgewiesen und kann auch selbst erprobt werden, wenn man einmal in vielen schwarzen und weißen Flecken den Dalmatiner erkannt hat, gelingt dies beim nächsten Versuch leichter. ${ }^{27}$

Wenn dies aber so ist, ist es umso wichtiger, sowohl entsprechendes Vorwissen über den „Stand der Kunst“ bezüglich eines Themas als auch über methodische Möglichkeiten, kreativ zu neuen Einsichten zu gelangen, zu vermitteln. Einführungen, Impulse und „propädeutische Übungen " sind daher wichtig, um den Potenzialraum zu erweitern. Hinzu kommen Übungen zur Imaginationsfähigkeit, wie Storytelling, Spiele, Improvisationstheater oder Science Fiction. ${ }^{28}$

Gegenüber dem menschlichen Bewusstsein, auf Seiten der „Realität“, geht es um neue Ansätze erfolgreicher Gestaltung (Design) von Innovationskontexten und Innovationsprozessen. Jedes Design löse Vorstellungsbilder und Erinnerungen aus, ausgedehnte Assoziationsräume, ganze Welten im Kopf. Dabei wirke Design besonders auf uns, wenn es an etwas an- körperliches Empfinden ${ }^{31}$, bis hin zu einem möglichen „Flow“-Zustand, in dem kreative Anstrengungen der Suche, Auseinandersetzung und Formgebung eher spielerisch ablaufen können. Dies hat Folgen für die Struktur sog. Innovation Labs, Barcamps oder Hackathons. ${ }^{32}$

Im Übrigen lassen sich Innovationen sowohl durch konvergentes als auch durch divergentes Denken entwickeln. ${ }^{33}$ Die Eingrenzung des Suchfeldes kann

\footnotetext{
22 Hill 2020, S. $211 \mathrm{f}$.

23 Daugherty/Wilson 2018, S. 146 ff., 151.

24 Christian/Griffiths 2020, S. 322; Hannes Federrath, Vortrag am 7. Februar 2020 in Speyer.

25 Mast 2020, S. 46.

26 Seth 2020, S. 20.

27 Wentura/Frings 2013, S. 65.

28 Hill 2020, S. 212.

29 Heimann/Schütz 2018, S. 20, 23.

30 Pabst, u. a. 2019, S. 47.

31 Heimann/Schütz 2018, S. 29.

32 Hill 2016a; Schmitz, u.a. 2020; Kohne/Wehmeier 2019; Krüger 2019.

33 Nielsen/Thurber 2018, S. 70 f.
} 
sinnvoll sein, um nicht vollständig im Dunkeln $\mathrm{zu}$ tappen. Visualisierungen durch Graphic Design oder durch Canvases $^{34}$ zur Verwesentlichung und Fokussierung der Ansätze können hilfreich sein. Umgekehrt ist auch der Zufall manchmal ein guter Helfer, indem Assoziationen zu völlig entfernt liegenden Begriffen gesucht werden oder durch zufällig gewählte Karten mit Bildern und Themen Assoziationen und neue Verknüpfungen hergestellt werden. ${ }^{35}$

\section{Innovationsprozess}

Für Innovationsprozesse gibt es verschiedene Anleitungen. Im Hinblick auf gesellschaftliche Innovationen schlagen Karl werkstätten in drei Phasen entwickelt hat. Danach wird in der Kritikphase die Frage gestellt: Was ist denn das Problem? Für die Phantasiephase heißt es: Wie wäre es, wenn's schön wäre? Für die Verwirklichungsphase lautet die Frage: Wie könnt's denn gehen ${ }^{38}$

Vanessa Burgardt vom Amt für kreative Problemlösungen in Leipzig sieht als Phasen Systematischer Kreativität: Vision formulieren, Situation erkunden, Herausforderungen formulieren, Ideen erkunden, Lösungen formulieren, Akzeptanz formulieren und Plan formulieren. ${ }^{39}$

Speziell für Innovationsworkshops gibt es ebenfalls verschiedene Anleitungen.

\section{"Bereits an anderer Stelle wurde darauf hingewiesen, dass die Gestaltung des Raums und die Zusammensetzung des Teams von großer Bedeutung sind."}

Peter Sprinkart, Franz-Theo Gottwald und Klaus Sailer folgende Phasen für den Innovationsprozess vor: Startphase (Impulse), Erkenntnisphase (Finden statt Suchen), Formungsphase (Perspektiven verändern), Modellierungsphase (Ideen für eine Innovation), Business-ModelingPhase (Architektur für die Lösung), Implementierungsphase (Vom Denken zum Handeln), Entwicklungs- und Wachstumsphase (Impact skalieren). ${ }^{36}$

Für soziale Transformationen verweisen die Autoren zudem auf die Methode „Transformative Scenario Planning“ nach Adam Kahane. Sie besteht aus den Schritten: Generieren eines Teams aus dem System, Analyse der aktuellen Situation des Systems, Entwickeln von Geschichten, Was getan werden müsste und Act to transform. ${ }^{37}$

Anregend ist nach wie vor die Methode, die Reinhard Sellnow für Zukunfts-
Manche sind an den inzwischen klassischen Design-Thinking-Prozess angelehnt: Verstehen, Beobachten, Sichtweise definieren, Ideen finden, Prototypen entwickeln und Testen. Da sich dieser Ansatz auf komplexe Problemstellungen beziehe und sich besonders für langandauernde Prozesse eigne, empfehlen Reinhold Pabst, Vera Podlinski und Lisa Koch vom Fraunhofer Institut für Angewandte Optik und Feinmechanik in Jena für den WorkshopKontext einen zusammengefassten Innovation Circle mit den Phasen: Initiieren, Generieren, Präsentieren und Evaluieren. ${ }^{40}$

Gerhard Embacher-Köhle von der Innovation Factory des Bundesrechenzentrums in Wien schlägt folgende Phasen vor:

- Warm-up: Einleitung und Erklärung der Methodik

- Inspiration Pitch: Input zum Thema und zur Motivation

- Visioning: Entwicklung einer Vision
- Discovery: Darstellung und Priorisierung von Use Cases

- Ideation: Ideengenerierung zu den drei wichtigsten zuvor entdeckten Use Cases

- Creation: Ausarbeitung von Abläufen und Interaktionen inclusive „Mockups"

- Wrap up \& Next Steps: Zusammenfassung und Ausblick ${ }^{41}$

Schon beim Inspiration Pitch, aber auch im gesamten Verlauf des Workshops ist es immer wieder wichtig, sich die Frage vor Augen zu halten, warum man etwas tut und für wen das wichtig ist. Methoden wie der Golden Circle oder das Value Proposition Design können dabei wichtige Unterstützung liefern. ${ }^{42}$

Bereits an anderer Stelle wurde darauf hingewiesen, dass die Gestaltung des Raums und die Zusammensetzung des Teams von großer Bedeutung sind. ${ }^{43}$ Auch die „Anwärmphase“ sollte nicht unterschätzt werden. Das betrifft sowohl die Vorbereitung und Hinführung der Individuen als auch der Gruppe zu einem innovativen Mindset und kreativen Klima. Dazu gibt es viele Anleitungen für gute Moderation und anregende Spiele ${ }^{44}$, die Offenheit, Vertrauen und Motivation schaffen sollen.

Die Hirnforschung und die Verhaltensforschung zeigen uns, dass gute Ideen wachsen und reifen müssen, Wiederholungen und Schleifen erforderlich sind, in denen der Potenzialraum ausgebaut und Verknüpfungen ermöglicht werden können. Insofern erscheint es als interessant und hilfreich, zu Beginn mit Begriffen und

\footnotetext{
34 Hill 2019a, S. 252.

5 Nielsen/Thurber 2018, S. $70 \mathrm{ff}$.

36 Sprinkart, et al. 2014, S. 128 ff.; vgl. noch Koschel/ Bösemüller 2017.

Kahane 2012; Sprinkart, u.a. 2014, S. $169 \mathrm{ff}$.

8 Sellnow 2012, S. 46 ff., 54 ff., $73 \mathrm{ff}$.

Burgardt 2019, S. 39.

Pabst, u.a. 2019, S. $77 \mathrm{f}$

Vortrag beim Besuch des Führungskollegs Speyer am 6. Juni 2019 in Wien.

42 Pabst, u.a. 2019, S. 159, 166; Hill 2019b, S. 979

43 Hill 2017, S. 272, 276.

44 Föhr 2018; Tonhauser 2018; Beermann, u.a. 2019.
} 
Bildern zu arbeiten, die zwar nicht direkt mit dem Problem, aber doch mit verwandten Inhalten zu tun haben. ${ }^{45}$

Pausen sind für Abschalten und Erholen wichtig. Die Konzeption eines Workshops über zwei Tage führt dazu, dass nachts auch das Unbewusste Blockaden lösen, sortieren und neu verknüpfen kann. Der aus der Psychologie bekannte Zeigarnik-Effekt zeigt, dass gerade Unerledigtes
Bereich der Kommunikationswissenschaft entwickelt worden. Dazu gehören: ${ }^{49}$

- Zeigen von Anwendungs- und Einsatzmöglichkeiten mithilfe von einfachen und plastischen Anwendungsbeispielen (Use Cases, Micro Frames, etc.)

- Verdeutlichen des Bezugs der Innovation zu aktuellen Themen. Hilfreich kann etwa die Verknüpfung mit aktuellen Herausforderungen, Trends oder zukünftigen Schlüsselereignissen sein. ${ }^{50}$
Mit einer entwickelten oder erfolgreich umgesetzten Innovation ist die Arbeit somit nicht abgeschlossen. Innovation bleibt eine Daueraufgabe, auch für den öffentlichen Sektor: Bleib innovativ, Verwaltung!

\section{"Von entscheidender Bedeutung ist die Frage, wie Innovationen kommuniziert und transferiert werden."}

eher zur Weiterbeschäftigung motiviert als eine abgeschlossene Einheit. ${ }^{46}$

Ein verbindlicher Leitfaden für Innovationsworkshops scheint nicht empfehlenswert. Vielmehr sollte mit Auftraggebern und Akteuren anhand der oben dargestellten oder ähnlicher Leitlinien jeweils abgestimmt werden, was für die jeweilige Herausforderung das beste Vorgehen darstellt.

\section{Innovationskommunikation und Innovationstransfer}

Innovationen sind nicht immer erwünscht. Sie bringen Veränderungen mit sich, machen Arbeit und verursachen zum Teil auch Ängste und Widerstände. Diese teilweise anzutreffende Skepsis oder gar Ablehnung betrifft auch die Personen, die sich mit Innovationen beschäftigen und diese vorschlagen. Sie werden manchmal eher als Störenfriede angesehen denn willkommen geheißen. ${ }^{47}$ Deshalb erscheint es ratsam, dass sie sich austauschen und wechselseitig auf ihrem Weg bestärken. ${ }^{48}$

Vor allem aber zeigt dies, dass die Frage, wie Innovationen kommuniziert und transferiert werden, von entscheidender Bedeutung ist. Einige Regeln sind dazu im
- Darstellung des konkreten Wertes der Innovation bzw. des Nutzens für die Anwenderinnen und Anwender, etwa mit typisierten Beispielen (Personas)

- Storytelling rund um die Innovation, etwa mit anschaulichen Erlebnissen und Erfahrungen.

Sowohl bei der internen als auch bei der externen Kommunikation kommt es dabei sehr auf die Darstellung und Präsentation, vor allem auf die Sprache an. Sie soll einfach, konkret, persönlich und aktivmotivierend $\operatorname{sein}^{51}$. Bekannt sind etwa Verknüpfungen, wie „Ja, und“ statt „Ja, aber“ oder positive Frames, wie „Herausforderung“ statt „Problem“. Auch wenn dies von manchen als Schönfärberei angesehen wird, werden aus der sprachlichen Darstellung subtile Botschaften abgeleitet, die Einstellung und Motivation beeinflussen können.

\footnotetext{
45 Pabst et al. 2019, S. 139.

46 Cialdini 2017, S. 109.

47 Hill 2019a, S. 254.

$48 \mathrm{Zu}$ sog. Communities of Practice vgl. schon Zboralski/Gemünden 2009.
}

\section{Literatur}

Beermann, S./Schubach, M./Tornow, O. (2019): Spiele für Workshops und Seminare, Freiburg im Breisgau.

Bormann, H.-W. /Benfer, M./Bormann, G. ( 2019): Change durch Co-Creation, Frankfurt/New York.

Borth, D. (2019): Kontrolle wie? oder Zum Aufbau einer sozialen Datenwirtschaft. In: Rodenstock, R./Sevsay-Tegethoff, N. (Hrsg.), 2020. Der Zukunftsnavigator, Roman-Herzog-Institut, Freiburg, S. 130-147.

Burgardt, V. (2019): Vom Wunschdenken zum Handlungsplan. In: Innovative Verwaltung, Heft 5, S. 38-40.

Christian, B./Griffiths, T. (2020): Algorithmen für den Alltag. Die Wissenschaft der perfekten Entscheidung - Vom Aufräumen bis zur Partnersuche, München.

Cialdini R. (2016): Pre-Suasion. Wie Sie bereits vor der Verhandlung gewinnen, New York.

Daugherty, P.R./Wilson, H.J. (2018): Human + Machine. Künstliche Intelligenz und die Zukunft der Arbeit, München.

Ebert, H./Münch, E. (2018): Sprache als Instrument der Change- und Innovationskommunikation, Wiesbaden.

Föhr, T. (2018): Moderationskompetenz für Führungskräfte. Methoden und Mindset für Meetings mit Partizipation, Eigenverantwortung und Kreativität, Bonn.

Fröhlich, R./Szyzka, P./Bentele, G. (Hrsg.) (2015): Handbuch der Public Relations. 3. Aufl., Wiesbaden, S. 965-975.

Harris, T. (2019): Our brains are no match for our technology. Unter: https://www.nytimes. com/2019/12/05/opinion/digital-technologybrain.html.

Heimann, M./Schütz, M. (2017): Wie Design wirkt. Psychologische Prinzipien erfolgreicher Gestaltung, Bonn. 
Hill, H. (2020): "Bounded Rationality“ im digitalen Zeitalter. In: DöV, Heft 6, S. 205-216.

Hill, H. (2019b): Die Kunst des Organisierens. In: DÖV, Heft 24, S. 973-981.

Hill, H. (2019a): Sei innovativ, Verwaltung! Weichenstellungen und Impulse zur Innovationsfindung, Verwaltung \& Management, Heft 6,5 . 251-263.

Hill, H. (2018b): Prüfung situativ-experimentellen Verwaltungshandelns. In: Deutsches Verwaltungsblatt, Heft 18, S. 1185-119o

Hill, H. (2018a): Agiles Verwaltungshandeln im Rechtsstaat. In: DÖV, Heft 13, S. 497-504.

Hill, H. (2016b): Start-Ups als Innovationspartner für den öffentlichen Sektor. In: Innovative Verwaltung, Heft 12, S. 8-10.

Hill, H. (2016a): Innovation Labs - Neue Wege zu Innovation im öffentlichen Sektor, DÖV Heft 12, S. 493-501.

Hill, H. (2014): Wandel von Verwaltungskultur und Kompetenzen im digitalen Zeitalter. In: DVBI, Heft 2, S. 85-93

Hill, H. (2013): Wandel-Galerie - Alte und neue Konzepte zum Change Management. In: Verwaltung und Management, Heft 5, S. 227-236.

Hill, H. (2011): Von Innovationsmanagement und Management der Unsicherheit zur zukunftsfähigen Verwaltung. In: Verwaltung und Management, Heft 1, S. 3-7.

Hill, H. (2006): Nachhaltige Verwaltungsmodernisierung. FÖV Discussion Papers 30.

Kahane, A. (2012): Working Together to change the future. Transformative scenario planning, San Francisco.

Kohne, A./Wehmeier, V. (2019): Hackathons. Von der Idee zur erfolgreichen Umsetzung, Wiesbaden.

Koschel, D./Bösemüller, D. (2017): Social Labs. In: OrganisationsEntwicklung, Heft 3, S. 91-96.

Kreimeier, N. (2017): Macht was! In: Capital, Heft 7, S. 26-38.

Kreimeier, N. (2018): Die netten Jahre sind vorbei. In: Capital, Heft 7, S. 26-29.

Krüger, N. (2019): Nerdig Working. In: Manager Seminare, Heft 259, S. 32-40.

Mast, C. (2019): Unternehmenskommunikation, München.

Mast, C. (2015): Innovationskommunikation. In: Fröhlich, Romy/Szyszka, Peter/Bentele, Günter (Hrsg.), Handbuch der Public Relations, 3. Aufl., Wiebaden, S. 965-975.

Mast, F. (2020): Black Mamba oder die Macht der Imagination. Wie unser Gehirn die Wirklichkeit bestimmt, Freiburg im Breisgau.

Nelke, A. (2019): Innovationskommunikation. In 30 Minuten wissen Sie mehr!, Offenbach.

Nielsen, D./Thurber, S. (2018): Die Kunst des kreativen Denkens. Assoziation, Inspiration, Idee, Berlin.

Pabst, R./Podlinski, V./Koch, L. (2019): Erfolgreiche Innnovationsworkshops. Zusammenarbeit individuell und kreativ gestalten, Weinheim.

Reinhardt, M. (2018): Agilität und Resilienz als starkes Duo im Digitalen Wandel. In: Gabal e. V. (Hrsg.), Impulse für den digitalen Wandel, S. 108-117.
Rettig, D. (2020): Das gesunde Maß. In: Ada, Heft 1, S. 74-81.

Rogl, M. (2019): Meine wichtigste Frage für 2020 lautet: Welche Skills brauchen wir, um zukunftsfähig zu sein? In: Rodenstock, R./ Sevsay-Tegethoff, N. (Hrsg.): 2020. Der Zukunftsnavigator, Freiburg, S. 222.

Roth, Gerhard (2019): Stark wie ? oder Zur Resilienz von Organisationen. In: Rodenstock, R./Sevsay-Tegethoff N.(Hrsg.): 2020. Der Zukunftsnavigator, Roman-Herzog-Institut, Freiburg, S. 94-113.

Schmitz, A.P./Beer, A./Fölsing, J. (2020): Barcamps - als Seismographen für emergente Veränderungen. In: OrganisationsEntwicklung, Heft 1, S. 85-93.

Schlumpf, H.-U. (2019): Dialog- und Lernkultur in Organisationen. Sinnorientierte Selbstorganisation durch EvoluCreation gestalten, Stuttgart.

Sellnow, R. (2012): Die mit den Problemen spielen. Ratgeber zur kreativen Problemlösung, 9. Auflage, Bonn.

Seth, A. K. (2020): Wahrnehmung. Unsere inneren Universen. In: Spektrum der Wissenschaft, 2, S. 18-24.

Sprinkart, K.P./Gottwald F.-T./Sailer K. (2014): Fair Business. Wie Social Entrepreneurs die Zukunft gestalten, Regensburg/Berlin.

Tonhauser, P. (2018): $66+1$ Warm-up die dich als Trainer unvergesslich machen, Norderstedt.

Wentura, D./Frings, C. (2013): Kognitive Psychologie (Basiswissen Psychologie), Wiesbaden

Zerfaß, A./Möslein, K. M. (2009): Kommunikation als Erfolgsfaktor im Innovationsmanagement. Strategien im Zeitalter der Open Innovation, Wiesbaden.
Verwaltungsreform durch Open Government?

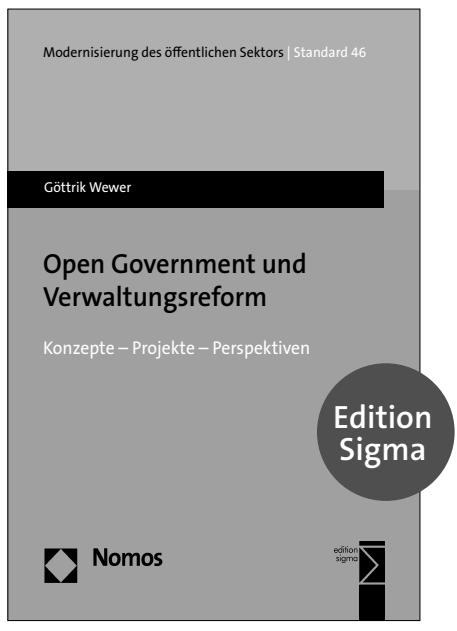

\section{Open Government und Verwaltungsreform}

Konzepte - Projekte -

Perspektiven

Von Dr. Göttrik Wewer

2020, 110 S., brosch., 29,-€

ISBN 978-3-8487-6787-8

(Modernisierung des öffentlichen

Sektors („Gelbe Reihe“), Bd. 46)

Ein offeneres Regieren und Verwalten soll die Behörden nicht nur besser machen, sondern dort auch einen Kulturwandel herbeiführen. Ob und wie Open Government in Bund, Ländern und Gemeinden als ein Instrument zur Modernisierung der Verwaltung verstanden wird, wird in dieser Studie untersucht. Dabei wird auch diskutiert, ob dieser Ansatz für die drei wichtigsten Stakeholder - Bürger Behördenmitarbeiter und Politiker-einen Mehrwert verspricht.

三ิ

Nomos 